\title{
REDE CAN VEICULAR: LEVANTAMENTO BIBLIOGRÁFICO E APRESENTAÇÃO DE CONCEITOS INICIAIS ${ }^{1}$
}

\author{
Andrey Gustavo de Souza ${ }^{2}$ \\ Gustavo Lobato Campos ${ }^{3}$
}

\begin{abstract}
RESUMO
O objetivo deste trabalho é apresentar as principais características de uma rede CAN veicular, tais como protocolos de camada superiores do modelo OSI, processo de transmissão de mensagens entre unidades no barramento CAN, formato de mensagens, tratamento de erro e também do processo de arbitragem. Uma breve apresentação sobre a rede CAN-FD - a evolução da rede CAN tradicional - é feita levando-se em conta as principais mudanças em relação a sua antecessora. É realizada uma avaliação considerando-se as vantagens do uso da atual tecnologia CAN empregada e os pontos fracos que essa topologia apresenta frente ao cenário do mercado atual, para, dessa forma, avaliar possíveis alternativas.
\end{abstract}

Palavras-chave: Rede CAN. CAN-FD. Redes automotivas. Protocolos de rede.

\section{INTRODUÇÃO}

Em meados da década de 1980, teve início o uso de centrais eletrônicas (ECU's), comumente conhecidas como nós ou módulos eletrônicos, com a finalidade de monitorar funções do veículo, controlar determinadas variáveis e prevenir uma série de incidentes indesejados. Dentre as funções que podem ser controladas por essas centrais, podem-se citar funções de segurança, como sistema de freios ABS, sistema de airbag, sensores diversos (sensor crepuscular, sensor de chuva, etc.), assim como funções do motor, como injeção eletrônica, bomba de combustível, monitoramento de temperatura, nível de óleo, entre outras. A operação incorreta de um desses elementos pode ocasionar falhas graves no veículo, que podem resultar na sua inutilização e até mesmo em acidentes.

\footnotetext{
${ }^{1}$ Como citar este artigo:

SOUZA, Andrey Gustavo de; CAMPOS, Gustavo Lobato. Rede can veicular: levantamento bibliográfico e apresentação de conceitos iniciais. ForScience: revista científica do IFMG, Formiga, v. 5, n. 1, e00234, jan.jun. 2017.

${ }^{2}$ Mestrando em Engenharia de Sistemas e Automação pela Universidade Federal de Lavras (UFLA). E-mail: andrey.souza@posgrad.ufla.br.

${ }^{3}$ Doutorando em Engenharia Nuclear pela Universidade Federal de Minas Gerais (UFMG) .Mestre em Engenharia Elétrica pela Pontifícia Universidade Católica de Minas Gerais (PUC-MG) na área de Compatibilidade Eletromagnética. E-mail: gustavo.lobato@ifmg.edu.br.
} 
SOUZA, A. G. de; CAMPOS, G. L.. Rede can veicular: levantamento bibliográfico e apresentação de conceitos iniciais

Com o aumento do número de funções veiculares ao longo do tempo, houve a necessidade de se conectar mais centrais eletrônicas e, ao mesmo tempo, simplificar o cabeamento sem comprometer a confiabilidade e a segurança do sistema.

Uma maneira de conectar essas centrais é por meio de uma arquitetura centralizada, como mostrado na FIG. 1, onde centrais, sensores e atuadores são conectados a um controlador central. Essa arquitetura resulta num sistema relativamente simples, onde os dados são armazenados em um controlador central (GUIMARÃES; SARAIVA, 2002), porém, ocasiona o aumento do cabeamento requerido, uma vez que cada elemento deve ser conectado ao controlador central, limitando e dificultando uma futura expansão do sistema.

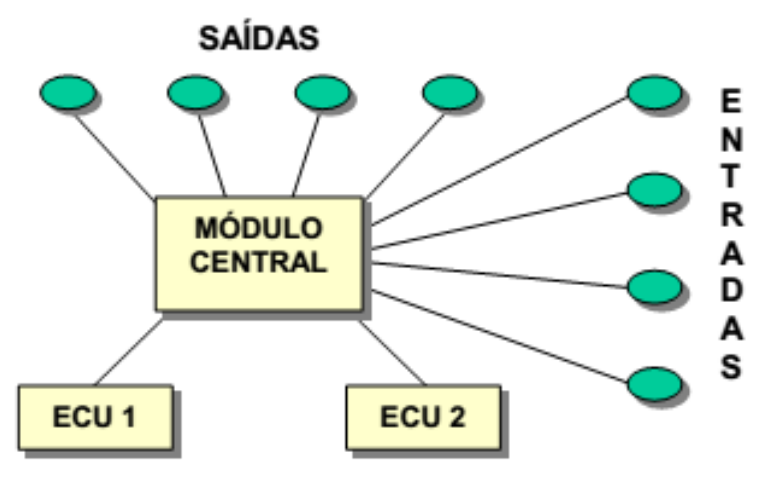

Figura 1 - Esquemático de uma arquitetura de comunicação veicular centralizada Fonte: Guimarães, Saraiva ( 2002).

Outra possibilidade é a arquitetura distribuída, como representado na FIG. 2, onde vários módulos estão distribuídos pelo veículo e cada uma dessas centrais recebe dados destinados a ela. Tal arquitetura resulta em um sistema mais simples, com cabeamento reduzido e com maior possibilidade de expansão quando comparado a sistemas automotivos de arquitetura centralizada. A comunicação entre centrais de um sistema de arquitetura distribuída é feita por intermédio de um software de controle de rede via protocolo de comunicação capaz de prover segurança e confiabilidade na troca de mensagens. 
SOUZA, A. G. de; CAMPOS, G. L.. Rede can veicular: levantamento bibliográfico e apresentação de conceitos iniciais

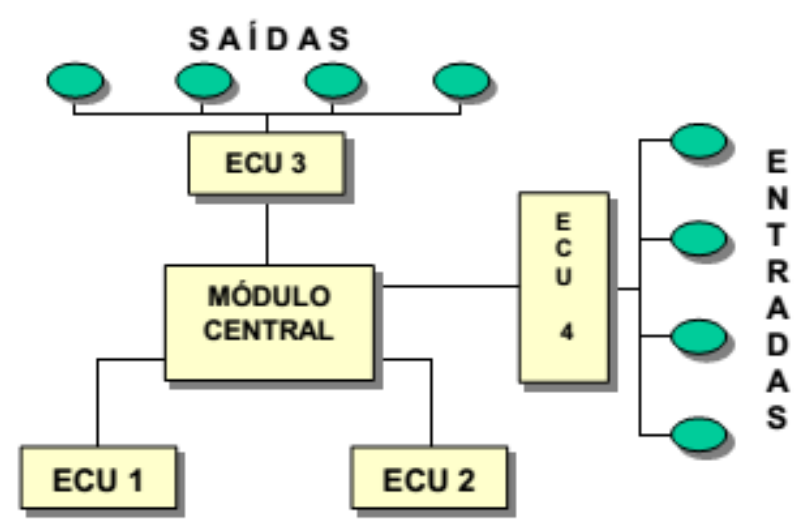

Figura 2 - Esquemático de uma arquitetura de comunicação veicular distribuída Fonte: Guimarães; Saraiva (2002).

A Aquitetura Distribuída é hoje a melhor solução onde existe a necessidade do uso de várias ECU's de controle de sistemas específicos. Porém, a forma como esses dados são transmitidos é definida por um protocolo de aplicações veiculares que deve possuir algumas características específicas, como robustez suficiente para garantir o funcionamento da rede, mesmo em ambientes nocivos; capacidade aprimorada de detecção de erros e falhas; expandibilidade; operar com informações em tempo real, entre outras funcionalidades desejadas (GUIMARÃES; SARAIVA, 2002).

Ciente dessas necessidades, o barramento Controller Area Network (CAN), como mostrado na FIG. 3, garante comunicação confiável, segura e eficiente entre sensores, atuadores, controladores e outros dispositivos de controle em tempo real. Assim, este artigo visa apresentar os principais conceitos pertinentes a esse tipo de rede, tais como os padrões de camadas superiores, em especial os de aplicações veiculares, assim como o formato de mensagens, formato de mensagem CAN, entre outros.

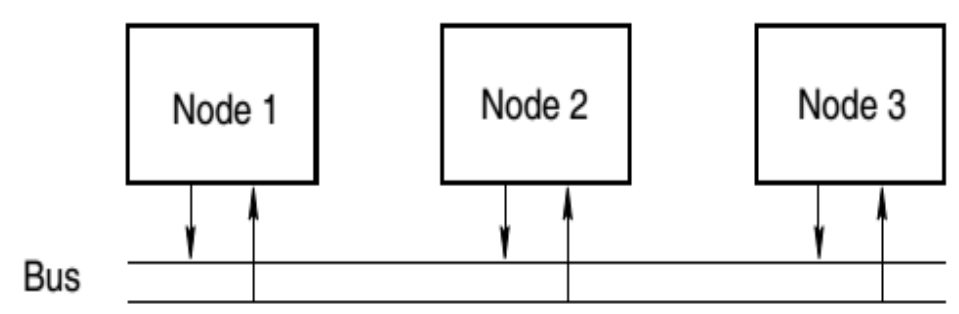

Figura 3 - Representação de nós conectados a um barramento CAN Fonte: Guimarães; Saraiva (2002).

\section{PROTOCOLOS DE CAMADA SUPERIOR}


SOUZA, A. G. de; CAMPOS, G. L.. Rede can veicular: levantamento bibliográfico e apresentação de conceitos iniciais

A rede CAN utiliza o protocolo de comunicação CSMA/CD+AMP (carrier-sense, multiple access protocol with detection and arbitration on message priority). O termo CSMA significa que cada nó do barramento deve esperar por um determinado momento de inatividade antes de tentar transmitir sua mensagem. $\mathrm{O}$ termo $\mathrm{CD}+\mathrm{AMP}$ significa que as colisões são solucionadas por meio de uma arbitragem orientada a bit, baseada numa prioridade de mensagem pré-programada no campo identificador da mensagem. Isto implica que todas as centrais verificam o status do barramento, conferindo se algum outro módulo não está transmitindo nenhuma mensagem com maior prioridade. Se o módulo que está transmitindo sua mensagem tem uma prioridade menor, esta transmissão é interrompida, e a de maior prioridade é transmitida.

O protocolo CAN padroniza as camadas físicas e de enlace do modelo OSI (DI NATALE et al., 2012), que são as duas camadas mais inferiores do modelo, como se pode observar na FIG. 4. Na maioria dos sistemas, as camadas superiores necessitam de outros protocolos para uma implementação e operação eficientes; porém, tais protocolos são os responsáveis por determinar como o protocolo CAN deve operar. Na maior parte dos casos em aplicações veiculares, somente algumas camadas (definidas de acordo com a necessidade da aplicação) acima das padronizadas pelo protocolo CAN são necessárias para operações em tempo real, sendo que mensagens redundantes não são contempladas pela normalização OSI (SOUSA, 2002).

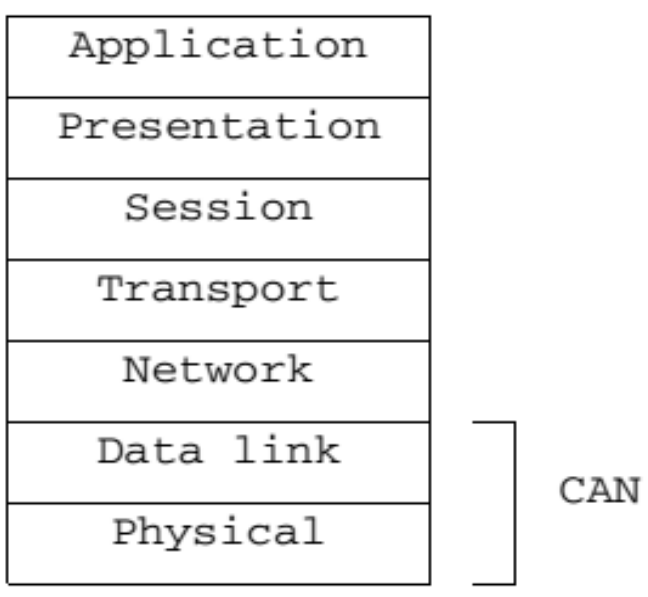

Figura 4 - Modelo OSI para protocolos de comunicação e as camadas abrangidas pela rede CAN

Fonte: Di Natale et al. (2012).

\subsection{CANOpen}


SOUZA, A. G. de; CAMPOS, G. L.. Rede can veicular: levantamento bibliográfico e apresentação de conceitos iniciais

O protocolo CANOpen define as cinco camadas superiores do modelo OSI. CANopen (BOTERENBROOD, 2000) é um protocolo que padroniza e implementa o uso da Rede CAN para dispositivos embarcados empregados em automação. Ele tem como características principais o gerenciamento de rede, o monitoramento de dispositivos e a comunicação entre nós. Outro ponto de destaque deste padrão é a possibilidade do uso de outros tipos de redes diferentes da CAN, dentre elas, a Ethernet.

\subsection{DeviceNet}

Outro protocolo que faz uso da rede CAN é o DeviceNet, sendo amplamente utilizado para a interligação de dispositivos de campo, tais como sensores, atuadores e controladores. Essa rede proporciona a interligação de até 64 nós endereçados de 0 a 63, denominado endereço MAC (Media Access Control). O endereço MAC é um endereço único, associado à interface de comunicação, que conecta um dispositivo à rede. Assim como o protocolo SAE J1939, a comunicação entre dispositivos é peer-to-peer, com prioridade e função de diagnóstico. Por ser um protocolo voltado para ambientes hostis, o DeviceNet é considerado um mecanismo de comunicação extremamente robusto a interferências eletromagnéticas (SEIXAS FILHO, [2007]).

\subsection{CAN Kingdom}

O protocolo CAN Kingdom, diferentemente dos outros protocolos de camada superior, não tenta seguir o modelo OSI. A rede, em sua maioria, é distribuída em nós, mas agora com a presença de uma central, ou nó mestre. Em suas especificações, o protocolo CAN Kingdom trata a rede em si como um reino, sendo a central principal a capital, e o restante, como cidades pertencentes a esse reino. O barramento CAN é tratado como a rota postal de mensagens entre as cidades. Seu funcionamento é simplificado, por não haver a necessidade do uso das camadas superiores, mas sim o uso de um conjunto de padrões primitivos (FREDRIKSSON, 1995). Uma comparação de uma rede CAN Kingdom com um barramento CAN tradicional é apresentada na FIG. 5, onde existe um módulo principal tratado como “capital”, e os módulos subordinados, como “cidades”. Já na rede CAN tradicional, não existe o conceito de módulo central e subordinados. 
SOUZA, A. G. de; CAMPOS, G. L.. Rede can veicular: levantamento bibliográfico e apresentação de conceitos iniciais

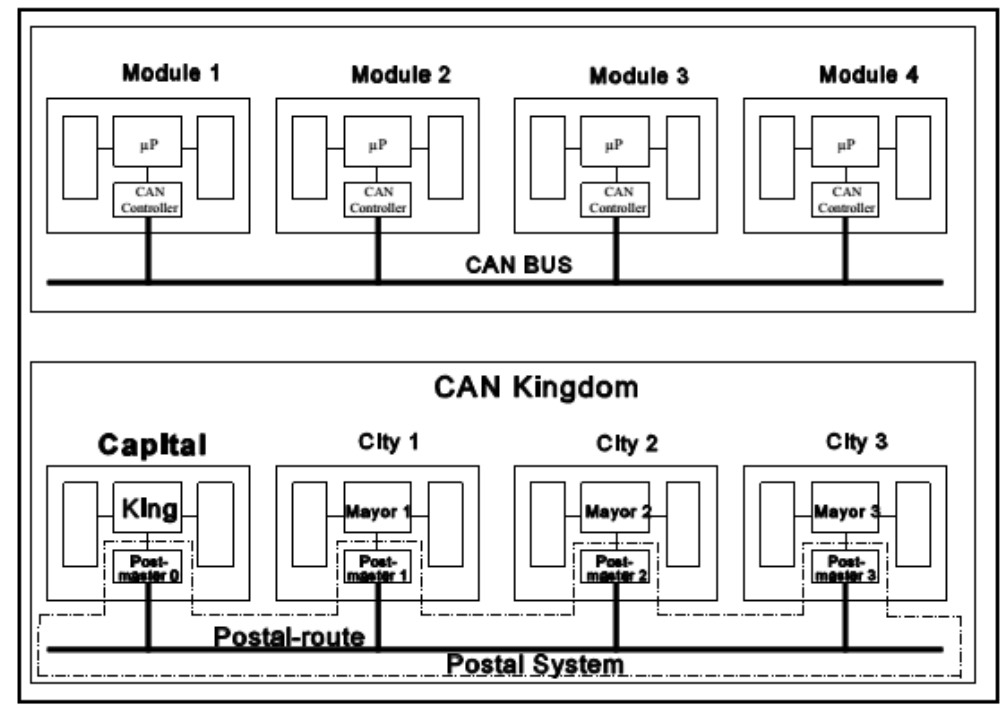

Figura 5 - Comparativo entre a rede CAN tradicional e o protocolo CAN Kingdom Fonte: Fredriksson (1995).

\subsection{SAE J1939}

Trata-se do protocolo mais usado em veículos onde diversos nós - ECUs - são conectados à Central Eletrônica. O protocolo SAE J1939 (JUNGER, 2010) define as cinco camadas superiores do modelo OSI e usa a rede CAN como camada física e de enlace. Sua prática recomenda como e quais dados são transmitidos entre as ECUs, dentro da rede. Tais centrais controlam, por exemplo, o motor, os freios, a transmissão, dentre outras várias possíveis funções.

O protocolo SAE J1939 possui taxa de transmissão média de $250 \mathrm{kbps}$, comunicação peer-to-peer (cada um dos nós da rede funciona tanto como cliente quanto como servidor) e por broadcast, gerenciamento de rede, através da arbitração de mensagens. Esse protocolo também proporciona uma definição de grupos de parâmetros para veículos comercias, como, por exemplo, a definição de quais mensagens têm maior prioridade. Além disso, conta com funções de diagnóstico, como a detecção e o tratamento de erro.

Existem diversos padrões derivados do SAE J1939, porém destinados a outros ramos, tais como o ISO11783, voltado para máquinas agrícolas, e NMEA2000®, para dispositivos marítimos, dentre vários outros. Na próxima seção, apresentar-se-á como a rede CAN opera com o protocolo SAE J1939. 
SOUZA, A. G. de; CAMPOS, G. L.. Rede can veicular: levantamento bibliográfico e apresentação de conceitos iniciais

\section{REDE CAN OPERANDO COM O PROTOCOLO SAE J1939}

O protocolo ISO-11898:2003 (este protocolo especifica as características de configuração de troca de informações entre módulos que utilizam a rede CAN) descreve como a informação é passada entre dispositivos em uma rede e padroniza as camadas física e de enlace CAN ao padrão OSI (ISO, 2015). Este protocolo trabalha um identificador de 11 ou 29 bits (a aplicação de cada um dependerá do número de mensagens necessárias no sistema a ser desenvolvido) e tem uma taxa de transmissão de $125 \mathrm{kbps}$ a 1 Mbps. O protocolo de 11 bits dispõe de $2^{11}$ mensagens diferentes, ou 2048 mensagens, Extented CAN, ou CAN Estendido, com identificador de 29 bits com 537 milhões de possibilidades de mensagens. Nas próximas subseções, serão apresentados os formatos de mensagens CAN 2.0A, de 11 bits, e CAN 2.0B, de 29 bits, além do formato do frame CAN.

\subsection{Protocolo CAN 2.0A com identificador de 11 bits}

A FIG. 6 mostra o formato de mensagem padrão da rede CAN 2.0A de 11 bits.

\begin{tabular}{|c|c|c|c|c|c|c|c|c|c|c|}
\hline S & 11-bit & R & I & & & & & & E & I \\
O & Identifier & T & D & r0 & D LC & 0...8 Bytes Data & CRC & ACK & O & F \\
F & & R & E & & & & & & F & S \\
\hline
\end{tabular}

Figura 6 - Padrão de mensagens da rede CAN de identificador de 11 bits Fonte: Corrigan (2002).

Cada um dos campos pode ser apresentado como:

- SOF: começo de quadro (start of frame) - é o bit que marca o início da mensagem. É usado para a sincronização dos nós após o barramento ficar inativo;

- Identifier: o padrão CAN com identificador de 11 bits estabelece a prioridade de cada mensagem, onde o menor valor binário do identificador indica maior prioridade para a mensagem;

- RTR: o bit de requerimento de transmissão remota. É dominante quando a informação é requerida por outro nó. Todos os nós recebem o requerimento, mas somente o identificador aponta a qual nó é destinada a mensagem. Isso proporciona uma uniformidade dos dados do sistema; 
SOUZA, A. G. de; CAMPOS, G. L.. Rede can veicular: levantamento bibliográfico e apresentação de conceitos iniciais

- IDE: bit identificador de extensão dominante (IDE) - especifica que uma mensagem CAN padrão (sem extensões no identificador) está sendo transmitida;

- r0: bit reservado para possíveis modificações futuras;

- DLC: esse campo de comprimentos de mensagens (4 bits) indica o número de bytes de dados que está sendo transmitido;

- Data: é a mensagem que se deseja transmitir, podendo chegar a 8 bytes (64bits);

- CRC: cyclic redundancy check (16 bits) - ou verificação de redundância cíclica contém o checksum da mensagem recém-transmitida, com função de detecção de erro;

- ACK: acknowledge error check - confirmação da checagem de erro. Cada nó que recebe uma mensagem precisa sobrescrever esse campo na mensagem original com um bit dominante, indicando que a mensagem recebida está livre de erro. Se um nó detectar um erro, ele descarta a mensagem e pede ao nó transmissor que a repita. Dessa forma, cada nó confirma a integridade da mensagem. ACK tem 2 bits, sendo o primeiro a confirmação, e o segundo, um espaço para o próximo campo;

- EOF: end of frame - esse campo de 7 bits indica o fim do quadro CAN e desabilita o preenchimento de bits, indicando um erro de preenchimento quando dominante;

- IFS: o espaçamento entre quadros - interframe space - contém o tempo requerido entre dois quadros.

\subsection{Protocolo CAN 2.0B Estendido com identificador de 29 bits}

O quadro padrão de mensagem do protocolo CAN 2.0B de 29 bits é indicado na FIG. 7:

\begin{tabular}{|c|c|c|c|c|c|c|c|c|c|c|c|}
\hline & $\begin{array}{c}\text { 11-bit } \\
\text { Identifier }\end{array}$ & $\begin{array}{l}\mathbf{S} \\
\mathbf{R} \\
\mathbf{R}\end{array}$ & $\begin{array}{l}\text { I } \\
\text { D } \\
\text { E }\end{array}$ & $\begin{array}{c}\text { 18-bit } \\
\text { Identifier }\end{array}$ & $\begin{array}{l}\mathrm{R} \\
\mathrm{T} \\
\mathrm{R}\end{array}$ & $1 \mathrm{r} 0$ & DLC & 0 ...8 Bytes Data & CRC & ACK & \begin{tabular}{|l|}
$\mathbf{E}$ \\
$\mathbf{0}$ \\
$\mathbf{F}$
\end{tabular} \\
\hline
\end{tabular}

Figura 7 - Padrão de mensagens da rede CAN de identificador de 29 bits Fonte: Corrigan (2002).

Esses 18 bits adicionais removem a limitação de mensagens encontrada no protocolo 2.0A. Como mostrado na FIG. 7, a mensagem do CAN estendido segue o mesmo protocolo do CAN tradicional, com a adição dos seguintes campos:

- SRR: bit de requerimento remoto substituto - substitui o campo RTR, como um placeholder (marcador de espaço) no formato estendido de mensagem; 
SOUZA, A. G. de; CAMPOS, G. L.. Rede can veicular: levantamento bibliográfico e apresentação de conceitos iniciais

- IDE: bit recessivo no campo de identificador de extensão - indica que os 18 bits adicionais no identificador virão a seguir. Os 18 bits identificadores vêm logo após esse campo;

- r1: assim como o campo r0, o bit r1 tem como função de espaço extra para futuras modificações no protocolo.

\section{PRINCIPAIS RECURSOS DA REDE CAN OPERANDO COM O PROTOCOLO SAE J1939}

A rede CAN, em operação conjunta com o protocolo de camada superior SAE J1939, dispõe de recursos interessantes, com a finalidade de garantir uma operação ágil, eficiente e confiável. Para isso, recursos como o de arbitragem de mensagens e diversos níveis de detecção e correção de erro são apresentados a seguir.

\subsection{Arbitragem}

A arbitragem é um mecanismo importante que trata dos conflitos de acesso no barramento (JOHANSSON; TÖRNGREN; NIELSEN, 2005). Quando o barramento CAN está livre, uma unidade inicia a transmissão de uma mensagem. Alguns possíveis conflitos podem existir devido à possibilidade de duas centrais iniciarem a transmissão simultaneamente. Esses tipos de conflito são resolvidos por meio de uma arbitragem orientada a bit, que define o nível de prioridade da mensagem contida no campo identifier em cada quadro transmitido.

Se os níveis de prioridade são iguais, a mensagem que está sendo transmitida continua sua transmissão normalmente. Quando a unidade detecta um nível dominante, enquanto uma mensagem não prioritária está sendo transmitida, a transmissão da mensagem menos importante é interrompida, e então a mensagem prioritária (aquela com o menor número binário) é enviada, e a ECU que estava transmitindo a mensagem passa a ser receptora (assim como todas as outras centrais presentes).

\subsection{Detecção e tratamento de erro}

Em um ambiente hostil e susceptível a interferências de diversas fontes, como um veículo, a prevenção de erros é uma parte essencial para garantir a confiabilidade, a precisão e a rapidez na resposta e na segurança do sistema. A detecção e o tratamento do erro são 
SOUZA, A. G. de; CAMPOS, G. L.. Rede can veicular: levantamento bibliográfico e apresentação de conceitos iniciais

características importantes no desempenho de uma rede CAN. Por causa de seus mecanismos complementares de detecção de erro, a probabilidade de haver um erro não detectado é pequena (JOHANSSON; TÖRNGREN; NIELSEN, 2005). Algumas das maiores vantagens do CAN é a sua robustez e a capacidade de contornar situações de falha temporárias ou permanentes. Pode-se classificar a detecção de erro de cinco diferentes maneiras, sendo três em nível de mensagem e dois em nível de bits. Os dois tipos de erro em nível de bit são:

- Monitoramento de bits: cada ECU presente na rede tem o papel de monitorar as mensagens disponíveis no barramento, e sinais de erro são transmitidos, caso seja detectada uma falha na mensagem por alguma unidade;

- Preenchimento de bits (bit stuffing): somente cinco bits consecutivos podem ter o mesmo valor, sendo este dominante ou recessivo. Se for necessária a transmissão de um sexto bit idêntico aos cinco anteriores, a unidade transmissora alocará um bit de valor contrário após cada conjunto de cinco bits idênticos e consecutivos. O módulo receptor fica a cargo de remover esse bit - o stuff bit. Caso a mensagem seja recebida com seis bits idênticos e consecutivos, algum erro ocorreu no barramento.

Em nível de mensagem, os três tipos de erro são:

- CRC (Cyclic Recundancy Check): trata-se de uma técnica checksum. O transmissor calcula o valor de acordo com os bits da mensagem e os transmite no campo CRC da mensagem. As unidades receptoras recalculam o CRC e realizam uma comparação com o valor recebido junto com a mensagem;

- Frame Check: as unidades receptoras analisam alguns bits da mensagem recebida. Esses bits são fixos (não mudam de mensagem para mensagem), e uma alteração neles (definidos pelo protocolo CAN) é indício de um erro;

- Acknowledgement Error Check (ACK): as unidades receptoras respondem cada mensagem íntegra recebida, escrevendo um bit dominante no campo ACK da mensagem, a qual é enviada de volta à unidade transmissora. Se esta mensagem de resposta não for recebida pelo transmissor da mensagem original, é sinal de que a mensagem transmitida estava corrompida, ou não foi recebida por nenhuma das unidades no barramento.

Todo e qualquer tipo de erro supracitado, quando detectado, acarretará em uma mensagem de erro no barramento, alertando toda a rede de que a mensagem anterior continha algum erro, obrigando o módulo transmissor a reenviá-la. 
SOUZA, A. G. de; CAMPOS, G. L.. Rede can veicular: levantamento bibliográfico e apresentação de conceitos iniciais

\section{A REDE CAN-FD}

A taxa de transmissão em torno de $1 \mathrm{Mbps}$ oferecida pela rede CAN padrão não é mais suficiente para atender a demanda de transmissão de dados dentro de um veículo. Uma solução para esse problema de largura de banda é usar o protocolo CAN com uma taxa de dados flexível (flexible data rate - FD) (HARTWICH, 2012). Essa versão aprimorada do protocolo CAN tradicional apresenta taxas de transmissão superiores a $8 \mathrm{Mbps}$ - o que foi possível graças a dois principais aprimoramentos (SAUERWALD, 2014):

- Dual bit rate: os quadros de mensagens do CAN-FD têm dois tempos diferentes para os bits de dados e os bits de cabeçalho e rodapé, e outro para o quadro de dados.

- Tempo de bit normal: o tempo de bit para os campos antes e depois dos dados de carga útil tem o mesmo tempo de bit que o protocolo CAN tradicional.

- Tempo de bit reduzido: a fim de se obter taxas de transmissões superiores, CAN-FD permite que alguns campos de uma mensagem tenham tempos de bits menores: o campo de dados, o campo de controle, o tamanho de mensagem e o campo CRC.

- O tamanho do campo de dados de carga útil tem tamanho variável, podendo chegar a 64 bytes -8 vezes maior que no protocolo CAN tradicional.

Observa-se que o requerimento de temporização é menos rígido no CAN-FD, uma vez que é garantido que os dispositivos transmitirão um após o outro. A arbitração orientada a bit não é necessária nesse caso.

\section{VANTAGENS E DESVANTAGENS DA REDE CAN}

A partir do momento em que o número de centrais eletrônicas presentes no veículo cresce, aumenta a necessidade de que o meio de comunicação entre as centrais também seja capaz de suportar tal demanda, que, muitas vezes, obriga o veículo a dispor de uma segunda rede, como, por exemplo, para suporte de conteúdo multimídia, que necessita de uma largura de banda maior para suportar transmissões de vídeo (JOHANSSON; TÖRNGREN; NIELSEN, 2005). A questão é que a necessidade de uma largura de banda maior geralmente não é um problema significativo, pois os elementos sensores, atuadores e ECUs não exigem tal esforço da topologia implementada. Na medida em que os veículos passam a dispor de sistemas de assistência, como câmera de ré, sistemas multimídia com interface ao usuário, entre outros, surge a necessidade de uma largura de banda superior. Como apresentado neste artigo, a rede 
SOUZA, A. G. de; CAMPOS, G. L.. Rede can veicular: levantamento bibliográfico e apresentação de conceitos iniciais

CAN sofre com tais limitações de largura de banda, o que torna comprometida a sua aplicação em determinados casos. Tais limitações trazem à tona a necessidade da busca por uma alternativa que apresente uma largura de banda superior, com a mesma confiabilidade e eficiência que a rede $\mathrm{CAN}$ provê.

Por outro lado, a rede CAN, em aplicações fundamentais do veículo, como controle do motor, transmissão, freios, dentre outras, funciona perfeitamente e garante uma operação eficiente, segura e robusta, mesmo em um ambiente hostil à transmissão de dados, sendo já considerado como uma opção o emprego de redes híbridas com o uso da rede CAN e a Ethernet automotiva para o suporte de centrais multimídias que requerem uma alta taxa de transferência de dados, sem abrir mão da confiabilidade que a rede CAN proporciona. Outro ponto forte da rede CAN é seu custo de implementação, pois, ano após ano, as vendas de equipamentos para esse tipo de rede têm aumentado, como apresentado na FIG. 8 (TUOHY et al., 2014).

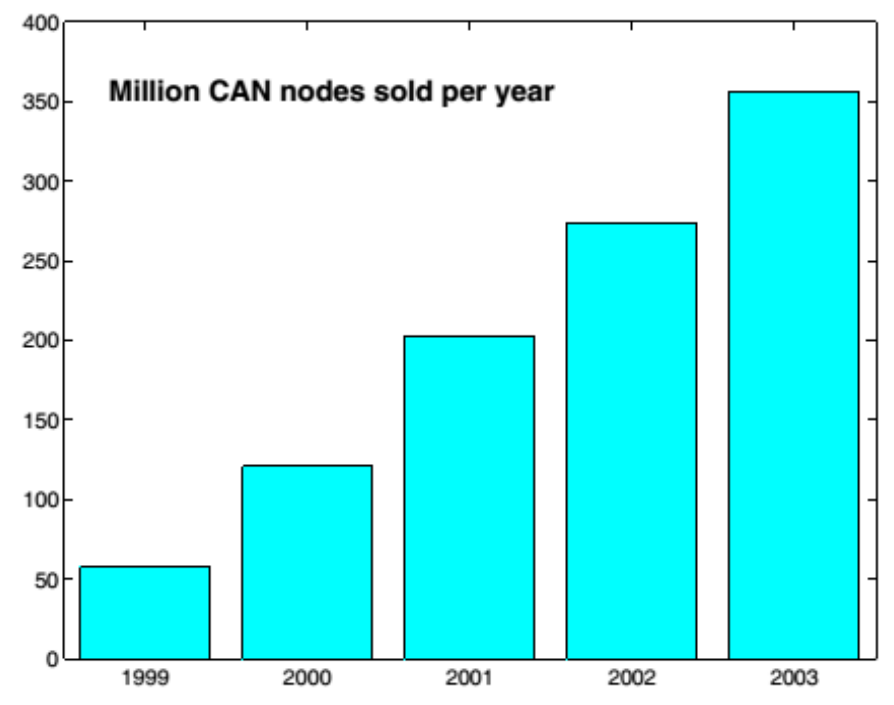

Figura 8 - Número de módulos CAN vendidos por ano Fonte: Association CAN in Automation (2004).

\section{CONSIDERAÇÕES FINAIS}

Este artigo teve como objetivo apesentar, de forma simplificada, as principais características da rede CAN veicular, bem como suas variantes destinadas a outros ramos (industrial, agrícola, navegação, entre outros). A forma como as mensagens são transmitidas pelo barramento CAN foi apresentada juntamente com a forma de tratamento de erro em cinco níveis (dois a nível de bit e três a nível de mensagem) e como isso faz com que a rede 
SOUZA, A. G. de; CAMPOS, G. L.. Rede can veicular: levantamento bibliográfico e apresentação de conceitos iniciais

CAN seja praticamente imune a mensagens errôneas. O sistema de arbitragem em 29 bits faz com que haja uma hierarquia bem definida das mensagens com maior prioridade e garante uma rápida resposta do veículo em caso de uma ocorrência mais grave.

Apesar de todas essas características, a rede CAN sofre com a baixa largura de banda, que limita sua aplicação em funções multimídias e tecnologias assistivas que demandam de uma operação streaming, em tempo real, e alta largura de banda. A implementação da rede CAN-FD, que proporciona taxas de transferência oito vezes maior que a rede CAN, torna-se paliativa, pois, apesar da notável evolução, os requerimentos do mercado em curto prazo não serão atendidos pela rede CAN, ou CAN-FD. Como trabalho futuro, tem-se a proposta de avaliar opções que atendam esses requerimentos e realizar um estudo comparativo e de viabilidade, tanto de implementação quanto econômica, de uma rede alternativa à rede CAN veicular.

\title{
AUTOMOTIVE CAN NETWORK: BIBLIOGRAPHICAL SURVEY AND PRESENTATION OF INITIAL CONCEPTS
}

\begin{abstract}
The objective of this document is to present the main features of a vehicular CAN network, such as upper layer protocols of the OSI model, the process of transmission of messages between the nodes in the bus, frame format, the error treatment and the priority arbitration of messages. A brief presentation about CAN-FD network, the evolution of traditional CAN network, is done considering the major changes from its predecessor. An evaluation is performed considering the advantages of its use and the weak features that this topology presents, considering the current market scenario, to thereby evaluate possible alternatives.
\end{abstract}

Keywords: CAN Network, CAN-FD. Automotive networks. Network protocols.

\section{REFERÊNCIAS}

BOTERENBROOD, H. CANopen high-level protocol for CAN-bus. NIKHEF, Amsterdam, Version 3.0, p. 1-23, Mar. 2000.

CORRIGAN, Steve. Introduction to the Controller Area Network (CAN). Application Report, Texas Instruments, Dallas (Texas), SLOA101, p. 1-15, 2002.

DI NATALE, Marco et al. Understanding and using the Controller Area Network communication protocol: theory and practice. New York: Springer, 2012.

FREDRIKSSON, Lars-Berno. A can kingdom. Mölndal, Sweden: Kvaser AB, 1995.

GUIMARÃES, Alexandre de Almeida; SARAIVA, Antônio Mauro. O protocolo CAN: entendendo e implementando uma rede de comunicação serial de dados baseada no 
SOUZA, A. G. de; CAMPOS, G. L.. Rede can veicular: levantamento bibliográfico e apresentação de conceitos iniciais

barramento “Controller Area Network”. Society of Automotive Engineers, p. 1-10, 2002.

HARTWICH, Florian. CAN with Flexible Data-Rate. INTERNATIONAL CAN

CONFERENCE (ICC), 3., 2012. Proceedings. Nuremberg (Germany), p. 14-1-14-9, 2012.

INTERNATIONAL ORGANIZATION FOR STANDARDIZATION. ISO 11898-1: Road vehicles: Controller area network (CAN): Part 1: Data link layer and physical signalling. Geneva, 2015. 65 p. Disponível em: <https://www.iso.org/standard/63648.html>. Acesso em: 15 jan. 2017.

JOHANSSON, Karl Henrik; TÖRNGREN, Martin; NIELSEN, Lars. Vehicle applications of controller area network. Hristu-Varsakelis, Dimitrios, Levine, William S. (Ed.). Handbook of Networked and Embedded Control Systems. College Park, MD: Birkhäuser Boston, 2005. p. 741-765.

JUNGER, Markus. Introduction to J1939. Vector, version 1.1, p. 1-11, 2010.

SAUERWALD, Mark. CAN bus, Ethernet, or FPD-Link: which is best for automotive communications? Analog Applications Journal, Texas Instruments, p. 20-22, 1Q 2014.

SEIXAS FILHO, Constantino. DeviceNet. Belo Horizonte: Departamento de Engenharia Eletrônica da UFMG, [2007]. 28 p. Apostila.

SOUSA, Rafael Vieira de. CAN (Controller Area Netwoork): uma abordagem para automação e controle na área agrícola. 2002. 83 f. Dissertação (Mestrado em Engenharia Mecânica) - Escola de Engenharia de São Carlos, Universidade de São Paulo, São Carlos, 2002. Disponível em: <http://www.teses.usp.br/teses/disponiveis/18/18135/tde-30102007115747/>. Acesso em: 15 jan. 2017.

TUOHY, Shane et al. Intra-Vehicle Networks : a review. IEEE Transactions On Intelligent Transportation Systems, v. 16, n. 2, p. 1-12 , Jan. 2014. DOI:10.1109/TITS.2014.2320605

Recebido em: 23/02/2017

Aprovado em: 27/06/2017

Publicado em: 19/07/2017 